\title{
Complicaciones del tratamiento quirúrgico del síndrome del túnel del carpo con el sistema "KnifeLight ${ }^{\circledR}$ "
}

\author{
F. J. García Bernal, A. Mora De Sambricio, J. Regalado, \\ R. Fuentes Gastañaga \\ Instituto Regalado y Bernal de Cirugía Plástica y de la Mano. Bilbao. España.
}

\begin{abstract}
Resumen: Los autores presentan una serie de complicaciones derivadas del uso del sistema "KnifeLigth ${ }^{\circledR}$,, instrumento diseñado para el tratamiento quirúrgico del síndrome del canal carpiano de una forma mínimamente invasiva.

Material y Método: Se presentan seis casos de complicaciones derivadas del uso del instrumental "KnifeLigth ${ }^{\circledR}$, , remitidos para tratamiento. Hubo dos casos de liberación incompleta, tratados mediante revisión quirúrgica e interposición con colgajo graso hipotenar, dos casos de lesiones parciales del mediano, tratadas de forma conservadora, un caso de sección de un nervio comisural tratado con injerto nervioso y colgajo graso hipotenar y una sección completa del nervio mediano reconstruida con injertos nerviosos, cobertura con vena autóloga y oponenteplastia.

Resultados: Todos los pacientes refirieron mejoría tras el tratamiento recibido, con un seguimiento mínimo de un año. Los casos de sección incompleta del ligamento anular del carpo evolucionaron como un caso primario estándar. Las secciones nerviosas reconstruidas con injertos nerviosos recuperaron sensibilidad de protección y experimentaron mejoría de la clínica de dolor.

Conclusiones: Si bien las técnicas mínimamente invasivas presentan algunas ventajas respecto a la técnica abierta, la severidad de las complicaciones derivadas de la falta de visión nos hacen ser reticentes a utilizarlas. Por otro lado, la interposición de grasa vascularizada o la cobertura con vena autóloga, constituyen técnicas de elección en cirugías secundarias de nervios.
\end{abstract}

Palabras clave: Síndrome del Túnel del carpiano, cirugía mínimamente invasiva, complicaciones intraoperatorias,

\begin{abstract}
The authors present a series of cases with complications arising from the use of "KnifeLigth", an instrument designed for the surgical treatment of carpal tunnel syndrome, as a mini-invasive technique.

Methods:We present six cases of complications of the surgical treatment of the carpal tunnel using the "KnifeLigth ${ }^{\circledR ”,}$, referred for treatment. There were two cases of incomplete release of the ligament, treated by surgical revision and an hypothenar fat flap interposition, two cases of partial injury of the median nerve treated conservatively, one case of common digital nerve division laceration treated with a nerve graft and an hypothenar fat flap, and one complete laceration of the median nerve reconstructed with nerve grafts, and an autologous vein coverage plus opponensplasty.

Results:All patients improved after the treatment with a minimum one year follow-up. The cases of incomplete section of the annular ligament carpal evolved as a standard primary case. The nerve lacerations reconstructed with nerve grafts recovered poor sensitivity and improvement of clinical pain.

Conclusions:While minimally invasive techniques have some advantages over the open technique, the severity of the complications due to the lack of vision make us reluctant to use them. Moreover, the interposition of fat or coverage with vascularized autologous vein, should be considered in secondary surgeries nerves.
\end{abstract}

Key words: carpal tunnel syndrome, minimal invasive surgery, intraoperative complications.

La compresión del nervio mediano en el canal carpiano o síndrome del túnel carpiano (STC), es la neuropatía compresiva más frecuente en extremidad superior, con una elevada prevalencia, entre 2.1 y 5.8 \% según las series, más frecuente en el sexo femenino ${ }^{1,2}$.

Ante el fracaso del tratamiento conservador está indicada la descompresión quirúrgica del nervio mediano. La liberación abierta del ligamento anular del carpo (LAC) es el tratamiento habitual de la neuropatía compresiva del canal carpiano. Gracias a una adecuada exposición y visión directa del ligamento, se reduce el riesgo de sección incompleta y lesiones iatrogénicas, permitiendo explorar el canal carpiano para descartar la existencia de patologías asociadas ${ }^{3}$.

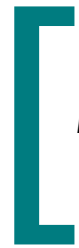


Desde la primera sección abierta del retináculo realizada por Galloway en 1924, se han publicado multitud de técnicas para la liberación del retináculo. En los últimos años se han extendido los abordajes mínimamente invasivos y la cirugía endoscópica, con el objetivo de reducir los inconvenientes secundarios al clásico abordaje abierto (cicatriz visible más o menos dolorosa, debilidad, recuperación funcional prolongada y dolor en los pilares tenar o hipotenar) ${ }^{4-7}$. No obstante, además de una larga curva de aprendizaje, estos abordajes mínimamente invasivos presentan una serie de inconvenientes y complicaciones (lesiones nerviosas o vasculares iatrogénicas y liberación incompleta) que han limitado su popularización ${ }^{8-1 !}$.

El "KnifeLight ${ }^{\circledR "}$ (Stryker Instruments, Kalamazooo, MI, USA) es un instrumento quirúrgico manual desechable, comercializado en 1998, diseñado para la liberación del LAC con visualización limitada. Mediante la incorporación de un sistema de iluminación integrada y gracias a su bisturí protegido entre dos láminas metálicas de borde romo, es posible seccionar el ligamento a través de una incisión de $1-2 \mathrm{~cm}$, de forma anterógrada o retrógrada, reduciendo así la afectación de los tejidos circundantes ${ }^{12}$.

El objetivo de este trabajo es mostrar una serie de complicaciones derivadas del uso del sistema "KnifeLigth ${ }^{\circledR}$ " y describir el tratamiento empleado para su resolución.

\section{MATERIAL Y MÉTODO}

En el periodo comprendido entre mayo de 2011 y enero de 2012, se revisaron seis casos de complicaciones secundarias al tratamiento quirúrgico del STC mediante el sistema KnifeLight ${ }^{\circledR}$, intervenidos en otros centros.

En los casos I y 2, los pacientes presentaban persistencia de la clínica por liberación incompleta. En los casos 3, 4 y 5 los pacientes sufrieron lesiones parciales del nervio mediano (dos con hipoestesia y uno con anestesia). En el caso 6, la paciente presentaba una sección completa del nervio mediano.

A continuación, se describen los distintos tipos de lesión y el tratamiento realizado.

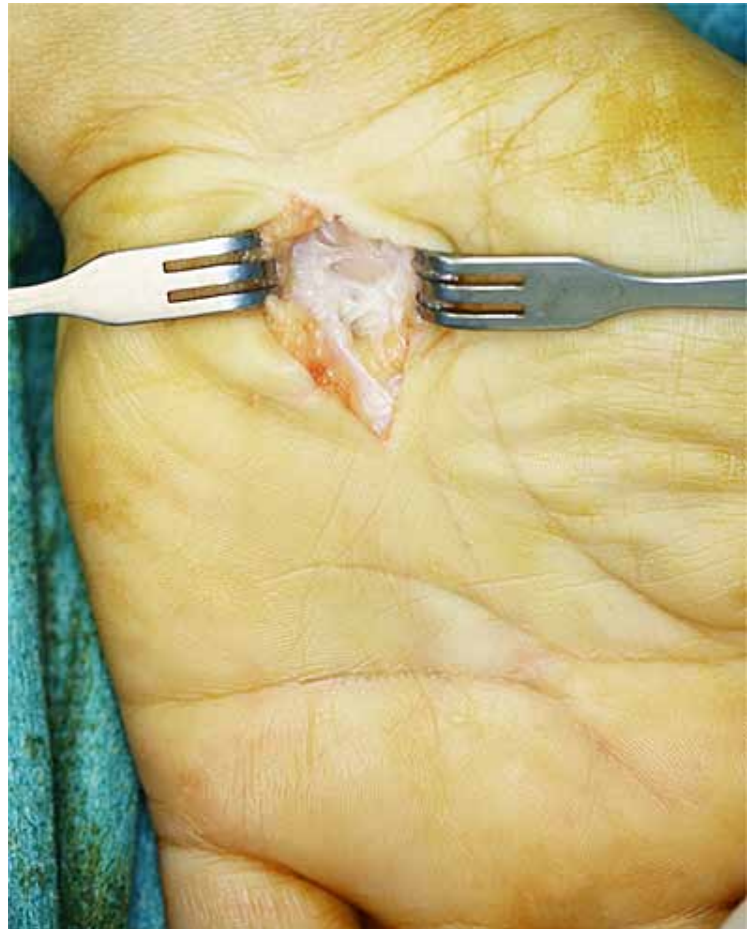

Figura I. Sección incompleta del retináculo flexor. Imagen intraoperatoria.

\section{Caso \| (sección incompleta del LAC)}

Paciente de 60 años, que acude a la consulta por persistencia de los síntomas de compresión del nervio mediano derecho, tras liberación quirúrgica del STC con KnifeLight ${ }^{\circledR}$, diez meses antes. Se realizó la exploración quirúrgica del canal carpiano a través de un abordaje palmar, apreciándose la apertura incompleta del retináculo flexor en su porción distal @ Figura I.Tras la sección del mismo, se procedió a interposición de un colgajo graso hipotenar (a) Figura 2 según la técnica descrita por Cramer en 1985'3.

\section{Caso 3 (lesión parciall del nervio mediano con hipoestesia)}

Paciente de 51 años intervenido de STC izquierdo, nueve meses antes. El paciente experimentó mejoría de la clínica de las parestesias nocturnas tras la intervención, pero refería disestesias en palma, a la altura del pliegue palmar proximal e hipoestesia en borde cubital del segundo dedo y radial del tercero. A la exploración presentaba un signo de Tinel positivo en pliegue palmar proximal, que gradualmente migró hacia distal con el paso de los meses. Fue tratado mediante rehabilitación con masaje local en la cicatriz. 


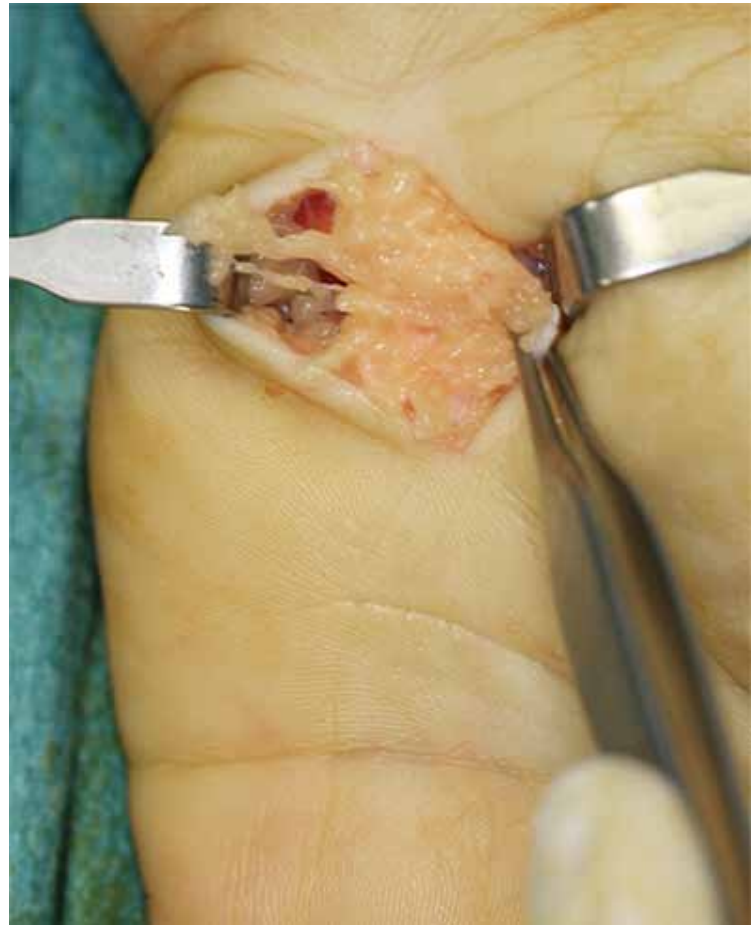

Figura 2. Colgajo de grasa hipotenar sobre canal carpiano tras la sección del ligamento.

\section{Caso 5 (lesión parcial del nervio mediano con anestesia)}

Paciente de 45 años, intervenido tres meses antes. La paciente refería haber mejorado la clínica de parestesias, pero a la exploración presentaba anestesia completa en borde cubital del primer dedo y radial del segundo, disestesia al contacto en la base de la eminencia tenar y un acusado signo de Tinel en la base de la eminencia tenar. En la revisión quirúrgica se apreció una laceración del primer nervio comisural @ Figura 3.Tras la reconstrucción con un injerto nervioso (aigura 4, se realizó la cobertura de la reparación nerviosa con un colgajo de grasa hipotenar.

Caso 6 (lesión completa del nervio mediano) Paciente de 33 años de edad, intervenida 6 meses antes con KnifeLight ${ }^{\circledR}$.A su llegada a nuestra consulta presentaba anestesia completa en el territorio del nervio mediano, imposibilidad para la oposición (M 0/5) y dolor secundario al neuroma. Se realizó una exploración quirúrgica en la que se comprobó una sección completa del nervio mediano (c) Figura 5. Tras la reconstrucción con injertos nerviosos, se protegió la reparación con injerto de vena autóloga (4) Figura 6 según técnica descrita por Sotereanos ${ }^{14}$ y se realizó una plastia de oposición con palmaris longus.

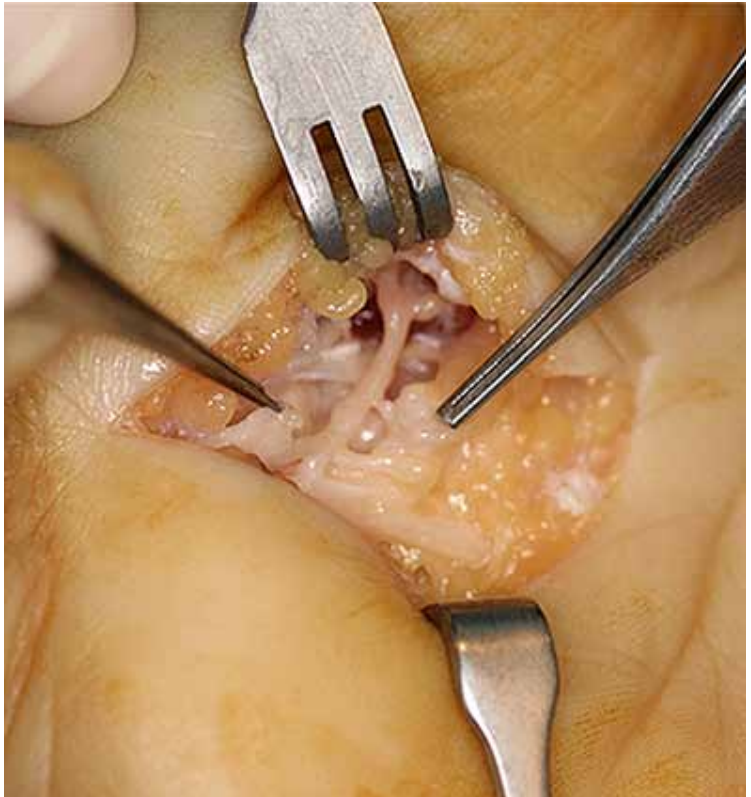

Figura 3. Laceración parcial del nervio mediano.

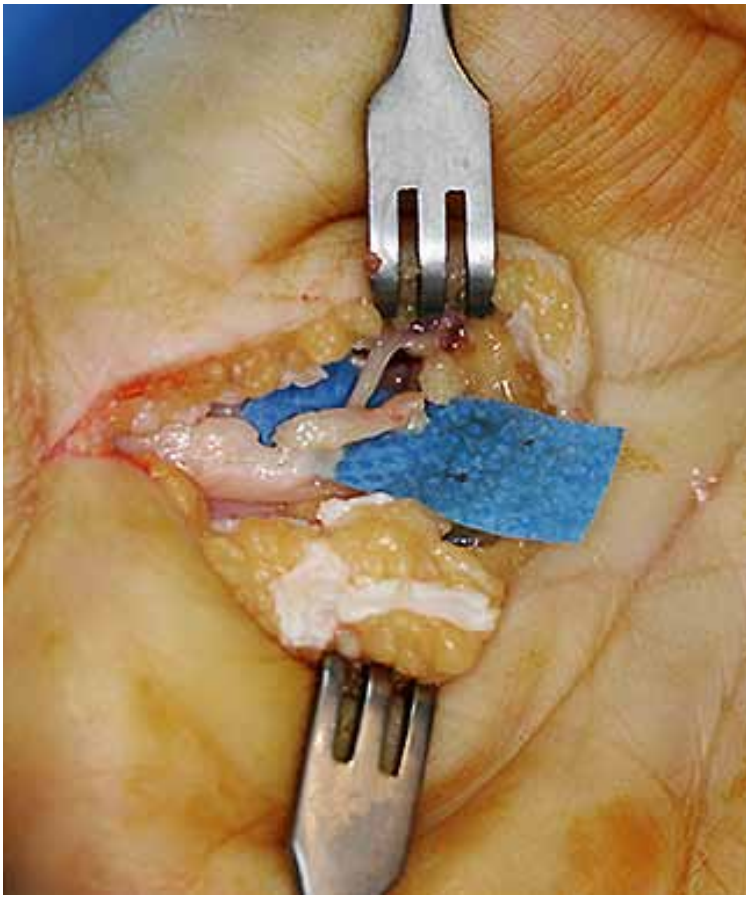

Figura 4. Injerto de nervio interóseo posterior previo a su sutura.

\section{RESULTADOS}

Con un seguimiento mínimo de un año, se evaluó el dolor según la Escala Visual Analógica (EVA) y la discriminación estática de dos puntos según la clasificación 


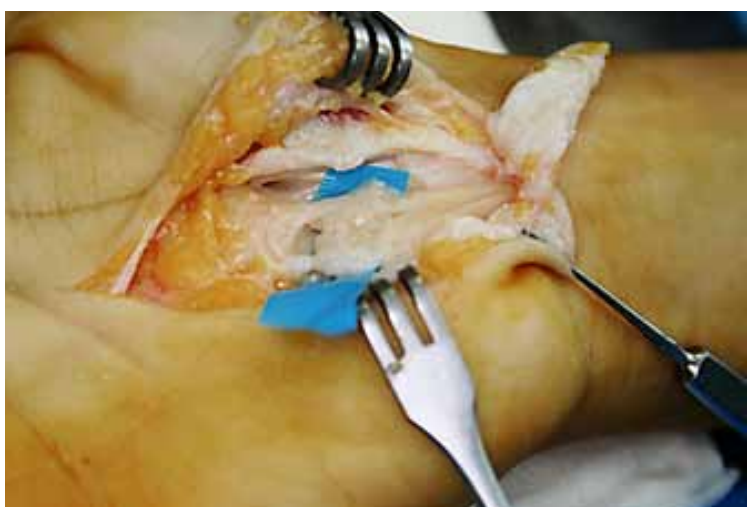

Figura. 5. Sección completa del nervio mediano.

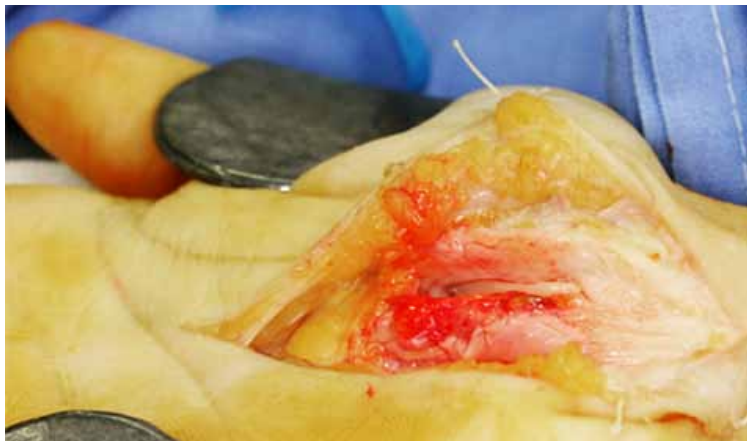

Figura 6. Cobertura de los injertos de nervio tras su sutura, con vena autóloga.

del "British Medical Research Council" modificada por MacKinnon y Dellon ${ }^{15,16}$. Los resultados se exponen en la (2) Tabla I. Los casos I y 2 , que presentaban una sección incompleta del LAC, evolucionaron de un modo similar a la de una liberación primaria estándar del túnel carpiano.
Los casos 3 y 4, con lesiones parciales del nervio mediano e hipoestesias, que fueron tratados con fisioterapia, evolucionaron en ambos casos, a la atenuación del signo de Tinel y mejoría de la clínica del dolor en el punto de irritación del nervio. El caso 3 experimentó una disminución de la puntuación de la EVA de 8 a I y el caso 4, de 7 a I. Por otro lado, la sensibilidad experimentó una mejoría limitada, con discriminaciones a dos puntos de $13 \mathrm{~mm}$ al año de seguimiento (caso 3) y II $\mathrm{mm}$ a los 14 meses (caso 4).

El caso 5, que había sufrido una sección de un nervio comisural, experimentó una notable mejoría del dolor en la eminencia tenar (disminución de la puntación de la EVA de 9 a I). Por el contrario, la recuperación sensitiva fue pobre (discriminación a dos puntos de $15 \mathrm{~mm}$ a los 15 meses de seguimiento).

La paciente con sección completa del nervio mediano, experimentó una mejoría sintomática del neuroma a los 15 meses de la reintervención con injerto nervioso (disminución de VAS de 9 a 2) y recuperación de la sensibilidad de protección con discriminación a dos puntos de $15-20 \mathrm{~mm}$ en el pulpejo de todos los dedos del territorio mediano, salvo el lado radial del índice, donde era de $25 \mathrm{~mm}$.

\section{DISCUSIÓN}

La neuropatía compresiva del nervio mediano a la altura del canal carpiano constituye una de las patologías más frecuentes en las consultas de cirugía de mano. Afecta frecuentemente a mujeres entre la quinta y la sexta décadas de la vida y ante el fracaso de las me-

\begin{tabular}{|c|c|c|c|c|c|c|c|c|}
\hline Caso $n^{\circ}$ & Género & Edad & Motivo de consulta & $\begin{array}{c}\text { Evolución } \\
\text { (meses) }\end{array}$ & $\begin{array}{l}\text { Hallazgos } \\
\text { operatorios }\end{array}$ & Tratamiento & $\begin{array}{c}\text { Seguimiento } \\
\text { (meses) }\end{array}$ & Resultado \\
\hline 1 & Mujer & 60 & Persistencia de síntomas & 10 & $\begin{array}{l}\text { Liberación } \\
\text { incompleta }\end{array}$ & $\begin{array}{l}\text { Apertura LAC + colgajo } \\
\text { de grasa hipotenar }\end{array}$ & 19 & $\begin{array}{c}\text { Curación } \\
2 \mathrm{pd}<6 \mathrm{~mm}(\mathrm{~S} 4) \text {, excelente * }\end{array}$ \\
\hline 2 & Mujer & 42 & Persistencia de síntomas & 7 & $\begin{array}{l}\text { Liberación } \\
\text { incompleta }\end{array}$ & $\begin{array}{l}\text { Apertura LAC + colgajo } \\
\text { de grasa hipotenar }\end{array}$ & 16 & $\begin{array}{c}\text { Curación } \\
2 p d<6 m m \text { (S4), excelente * }\end{array}$ \\
\hline 3 & Varón & 51 & $\begin{array}{c}\text { Tinel en palma } \\
\text { Hipostesia borde cubital del índice } \\
\text { y radial del } 3^{\text {er }} \text { dedo }\end{array}$ & 9 & $\begin{array}{l}\text { Lesión parcial } \\
\text { del N. mediano }\end{array}$ & Conservador & 13 & $\begin{array}{c}\text { Atenuación Tinel } \\
\text { Mejoría del dolor (VAS } 8 \text { a 1) } \\
\text { 2pd 13mm (S3+), bueno * }\end{array}$ \\
\hline 4 & Mujer & 41 & $\begin{array}{l}\text { Tinel en palma } \\
\text { Hipostesia lado cubital del } 3^{\text {er }} \text { dedo }\end{array}$ & 6 & $\begin{array}{l}\text { Lesión parcial del } \\
\text { N. mediano }\end{array}$ & Conservador & 14 & $\begin{array}{c}\text { Atenuación Tinel } \\
\text { Mejoría del dolor (VAS } 7 \text { a 0,5) } \\
\text { 2pd 11mm (S3+), bueno * }\end{array}$ \\
\hline 5 & Mujer & 45 & $\begin{array}{c}\text { Tinel en eminencia tenar } \\
\text { Anestesia completa lado cubital del } \\
\text { pulgar y radial del índice }\end{array}$ & 3 & $\begin{array}{c}\text { Sección de } \\
\text { nervio comisural }\end{array}$ & $\begin{array}{l}\text { Injerto nervioso con IOP + colgajo } \\
\text { de grasa hipotenar }\end{array}$ & 15 & $\begin{array}{c}\text { Atenuación Tinel } \\
\text { Mejoría del dolor (VAS 9 a 1) } \\
\text { 2pd 15mm (S3), pobre * }\end{array}$ \\
\hline 6 & Mujer & 33 & $\begin{array}{l}\text { Anetesia territorio mediano } \\
\text { Ausencia oposición (M 0/5) }\end{array}$ & 6 & $\begin{array}{c}\text { Sección completa } \\
\text { del N. mediano }\end{array}$ & $\begin{array}{l}\text { Injerto nervioso } \\
\text { Vein wrapping } \\
\text { Oponente-plastia }\end{array}$ & 15 & $\begin{array}{c}\text { Atenuación Tinel } \\
\text { 2pd 15-20mm excepto en lado radial } \\
\text { del índice } 25 \mathrm{~mm} \text { (S3), pobre }\end{array}$ \\
\hline
\end{tabular}

Tabla 1. * Según la clasificación del British Medical Research Council modificada por Mackinnon y Dellon's. 
didas conservadoras, la descompresión quirúrgica del nervio mediano es el tratamiento de elección ${ }^{17}$.

Si bien existen distintos abordajes, la sección del LAC a cielo abierto a través de un abordaje palmar, es la técnica más habitualmente empleada. Este abordaje reduce las complicaciones derivadas de una exposición insuficiente, como son las lesiones iatrogénicas del nervio mediano, de los arcos vasculares o la apertura incompleta del retináculo. Así mismo, permite explorar el canal carpiano y descartar la presencia de lesiones asociadas al STC.

Los inconvenientes de esta técnica, derivan de la presencia de una cicatriz en la palma, más o menos visible y dolorosa, la debilidad residual o el retraso en la recuperación de la fuerza y el riesgo de dolor en los pilares tenar o hipotenar.Tal es así, que la tasa de complicaciones del tratamiento quirúrgico del STC oscila entre un $3 \%$ y un $25 \%$ según autores ${ }^{18,19}$.

Con la finalidad de reducir esta morbilidad, aparecieron en la década de los 90 las técnicas mínimamente invasivas. La más popular fue la técnica endoscópica descrita por Chow ${ }^{20}$ en 1989, cuyo objetivo es reducir la longitud de la cicatriz y las molestias postoperatorias. Según los autores, al no lesionar el tejido subcutáneo, se reduce también el riesgo de dolor pilar y disminuye la debilidad residual, lo que repercute en una reincorporación más precoz al mundo laboral ${ }^{21}$. No obstante, como toda técnica nueva, la endoscópica requería una curva de aprendizaje, no exenta de complicaciones (lesiones nerviosas, vasculares, sección incompleta del ligamento anular del carpo). Por otro lado, se encarecía el procedimiento, por el incremento del tiempo quirúrgico y del instrumental necesario, motivos por los que no fue universalmente aceptado ${ }^{22}$.

En la línea de los abordajes mínimamente invasivos, apareció el instrumento KnifeLight ${ }^{\circledR}$, aprobado por la FDA en 1996 y comercializado a partir de 1998 por Stryker, aunque los primeros resultados no fueron publicados hasta $2000^{12}$. Su diseño permite la sección del ligamento anular del carpo a través de un abordaje mínimo, y gracias a la incorporación de un sistema de iluminación y una hoja de bisturí protegida, semeja en la forma de trabajo a la endoscopia, con un menor coste. Los inconvenientes de esta técnica vienen dados por el hecho de dejar una parte de la cirugía sin control visual, causa principal de las complicaciones comentadas en este artículo 6, 12,23-29.
No obstante, existen diversas publicaciones en las que se exponen los beneficios del tratamiento quirúrgico del túnel carpiano con esta técnica. Las ventajas atribuidas al KnifeLight ${ }^{\circledR}$ son la facilidad de su uso, la reducción de la incidencia de dolor en los pilares tenar e hipotenar y de molestias en la cicatriz y una reincorporación más precoz a la actividad laboral. En esta línea, algunos autores, definen la técnica como parcialmente "bajo visión directa"23,24.

Bhattacharya compara en su artículo la técnica abierta y el KnifeLight ${ }^{\circledR 24}$. Entre los casos tratados con el KnifeLight ${ }^{\circledR}$, sólo se describe un caso de parestesias en eminencia tenar, frente a ninguno en la serie sometida a cirugía abierta, sin diferencias en lo referente al dolor postoperatorio entre los dos grupos. Por otro lado, observan una menor incidencia de molestias en la cicatriz a las seis semanas con el KnifeLight ${ }^{\circledR}(8 / 26$ frente a 17/26 en la técnica abierta).

En un periodo inferior a un año, hemos recibido en nuestra consulta seis complicaciones debidas a la falta de control visual durante la sección del ligamento anular del carpo con el KnifeLigth ${ }^{\circledast}$. Dos casos eran aperturas incompletas, que tras la liberación por el abordaje palmar habitual, evolucionaron del mismo modo que un STC primario. De los tres casos con lesiones parciales del nervio mediano, los dos pacientes con hipoestesia fueron tratados de forma conservadora con masajes locales, experimentando una mejoría con el paso del tiempo. El caso de la anestesia por sección de nervio comisural fue tratado mediante reconstrucción con injerto nervioso.

Una vez los nervios son sometidos a revisiones quirúrgicas o múltiples intervenciones, la cicatriz alrededor del mismo puede ser causa de compresión o de formación de adherencias y por tanto de dolor e impotencia funcional ${ }^{30}$ De ahí que esté recomendada por múltiples autores, la interposición de tejido entre el epineuro y el entorno cicatricial| ${ }^{14,30}$. Siguiendo esta línea, en los casos descritos anteriormente, hemos empleado el colgajo de grasa hipotenar descrito por Cramer en 1985. La grasa hipotenar está irrigada por ramas perforantes de la arteria cubital a la altura del canal de Guyon y se encuentra en la vecindad del canal carpiano. Este colgajo es fácil de disecar, de movilizar y no precisa de cicatrices añadidas $13,18,19$. El inconveniente viene dado por la limitada cantidad de tejido que aporta.

Tal es así, que en aquellas situaciones donde el área del nervio a proteger es mayor, preferimos recubrir el 


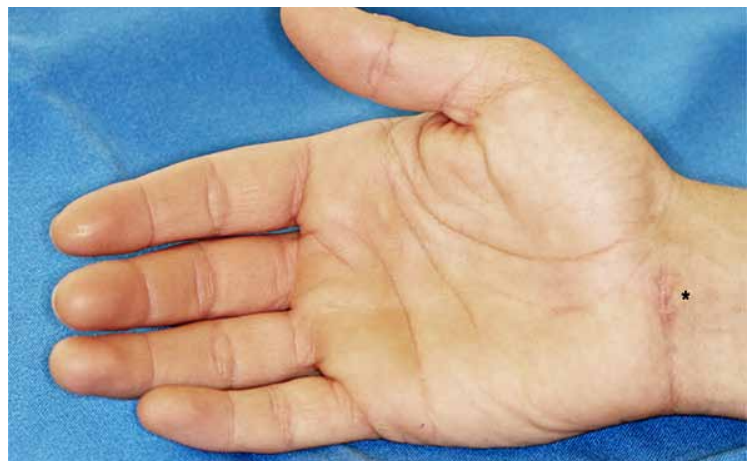

Figura 7. El punto negro señala la cicatriz del abordaje proximal realizado para el tratamiento con KnifeLight ${ }^{\circledR}$.

nervio con vena autóloga, siguiendo la técnica de Sotereanos ${ }^{14}$, como en el caso de la sección completa del nervio mediano reconstruida con injertos nerviosos. En este caso, ante la localización más distal de la reconstrucción nerviosa y el insuficiente tejido aportado por el colgajo de grasa hipotenar, optamos por la técnica de Sotereanos para aislar el entorno cicatricial de la reparación nerviosa.

Si bien existen múltiples técnicas para tratar las secuelas derivadas de las complicaciones del tratamiento quirúrgico del STC, su explicación o discusión excede el objetivo de este trabajo, que es el de alertar de las posibles complicaciones del tratamiento con esta técnica.

Las complicaciones que presentaban los pacientes de nuestra serie podrían ser atribuidas a un abordaje excesivamente proximal (2) Figura 7 con limitada visión en el momento de la sección del ligamento. Consideramos que el abordaje abierto habitual no precisa de una curva de aprendizaje tan "cara" para los pacientes $y$, por tanto, no somos partidarios de abordajes con visión limitada para el tratamiento del STC. Además, el KnifeLigth ${ }^{\circledR}$ es un instrumento desechable, lo que supone un coste adicional frente a la técnica abierta ${ }^{25}$.

No se ha incluido en este estudio los resultados de los estudios neurofisiológicos, por dos motivos. En primer lugar, por las conocidas limitaciones de esta prueba ${ }^{31}, y$ en segundo lugar, la diversa procedencia de los pacientes de diferentes centros, hacía cuestionable la fiabilidad interobservador.

\section{CONCLUSIÓN}

En la actualidad, la cirugía abierta del STC sigue siendo el tratamiento habitual en la mayoría de los centros.
Las técnicas mínimamente invasivas presentan ciertas ventajas frente a la técnica abierta, como una menor morbilidad y una reincorporación a la actividad habitual más precoz. Sin embargo, la magnitud de las complicaciones potenciales derivadas de la visualización limitada de estas técnicas, nos hacen ser escépticos respecto al uso de este tipo de instrumentos en la práctica clínica.

\section{AGRADECIMIENTOS}

A Maite Rodríguez Fernández, bibliotecaria del Hospital Universitario de Basurto, por su ayuda en la búsqueda bibliográfica.

\section{CONFLICTO DE INTERESES}

Los autores declaran no tener conflictos de intereses.

\section{BIBLIOGRAFÍA}

I. Latinovic R, Gulliford MC, Hugues RA. Incidence of common compressive neuropathies in primary care. J Neurol Neurosurg Psychiatry. 2006;77:2635.

2. Shiri R, Miranda H, Heliövaara M, Viikari-Juntura E. Physical work load factors and carpal tunnel syndrome: a population-based study. Occup Environ Med. 2009;66:368-73.

3. Mackinnon SE, Novak CB. Compression Neuropathies. En:Wolfe SW, Hotchkiss RN, Pederson WC, Kozin SH (Ed). Green's Operative Hand Surgery. $6^{a}$ ed. Philadelphia: Elsevier Churchill-Livingstone; 20I I.p. 977-10I4.

4. Chow JCY. Endoscopic release of the carpal ligament: a new technique for carpal tunnel syndrome. Arthroscopy. 1989;5:19-24.

5. Agee JM, McCarroll HR Jr, Tortosa RD, Berry DA, Szabo RM, Peimer CA. Endoscopic release of the carpal tunnel: a randomized prospective multicenter study. J Hand Surg Am. 1992; 17:987-95.

6. Cellocco P, Rossi C, El Boustany S, Di Tanna GL, Costanzo G. Minimally invasive carpal tunnel release. Orthop Clin North Am. 2009:40:44I -8.

7. Abouzar MK, PAtsis MC, Chiu DTW. Carpal tunnel release using limited direct vision. Plastic Recons Surg. 1995:534-8.

8. Chapman CB, Ristic S, Rosenwasser MP. Complete median nerve transection as a complication of car- 
pal tunnel release with a carpal tunnel tome. Am J Orthop. 2001;30:652-3.

9. Kernt B, Neu J. Injury to the median nerve after minimally invasive decompression: discrepancy between the surgical report and actual course of surgery. Unfallchirurg. 201 I; I 4:538-40.

10. Atik TL, Smith B, Baratz ME. Risk of neurovascular injury with limited-open carpal tunnel release: refining the "safe-zone". J Hand Surg Br. 2001;26:484-7.

I I. Shinya K, Lanzetta M, Conolly WB. Risk and complications in endoscopic carpal tunnel release. J Hand Surg Br. 1995;20:222-7.

12. Avci S, Sayli U. Carpal tunnel release using a short palmar incision and a new knife. J Hand Surg Br, 2000; 25(4): 357-60.

13. Cramer LM. Local fat coverage for the median nerve. En: Lanford LL (ed). Correspondence Newsletter for Hand Surgery. Chicago, IL: ASSH; 1985. p. 35.

14. Sotereanos DG, Giannakopoulos PN, Mitsionis $\mathrm{Gl}, \mathrm{Xu}$ J, Herndon $\mathrm{H}$. Vein graft wrapping for the treatment of recurrent compression of the median nerve. Microsurgery. 1995; 16:752-6.

15. Meek MF, Coert JH. Recovery of two-point discrimination function after digital nerve repair in the hand using resorbable FDA- and CE-approved nerve conduits. J Plast Reconstr Aesthet Surg. 2013;66(10): | 307-15.

16. Rosenfield J, Paksima N. Peripheral nerve injuries and repair in the upper extremity. Bull Hosp Jt Dis. 200 I-2002;60(3-4): | 55-6|

17. Huisstede BM, Hoogvliet P, Randsdorp MS, Glerum S, van Middelkoop M, Koes BW. Carpal tunnel syndrome. Part I: Effectiveness of nonsurgical treatments. A systematic review. Arch Phys Med Rehabil. 2010;91:981-1004.

18. Craft RO, Duncan SFM, Smith AA. Management of recurrent carpal tunnel syndrome with microneurolysis and the hypothenar fat pad flap. Hand. 2007;2:85-9.

19. Strickland JW, Idler RS, Lourie GM, Plancher KD. The hypothenar fat pad flap for management of recalcitrant carpal tunnel syndrome. J Hand Surg. 1996;2 | A:840-8.
20. Chow JC. Endoscopic release of the carpal ligament: a new technique for carpal tunnel syndrome. Arthroscopy. 1989;5: 19-24.

2I. Agee JM, McCarroll HR, North ER. Endoscopic carpal tunnel release using the single proximal incision technique. Hand Clin. 1994; 10:647-59.

22. Boeckstyns MEH, Sorensen Al. Does endoscopic carpal tunnel release have a higher rate of complications than open tunnel release? J Hand Surg Br. 1999;24B:9-15.

23. Helm RH,Vaziri S. Evaluation of carpal tunnel release using the KnifeLight instrument. J Hand Surg Br. 2003;28:25 I-4.

24. Bhattacharya R, Birdsall PD, Finn P, Stothard J. A randomized controlled trial of KnifeLight and open carpal tunnel release. J Hand Surg Br. 2004;29: I I35.

25. Hwang PY, Ho CL. Minimally invasive carpal tunnel decompression using the KnifeLight. Neurosurgery. 2007;60(2 Suppl 1):ONS I 62-8;discussion ONSI 68-9.

26. Yeo KQ, Yeo EM. Comparison of the results of open carpal tunnel release and KnifeLight carpal tunnel release. Singapore Med J. 2007;48: I I 3 I-5.

27. Teh KK, Ng ES, Choon DS. Mini open carpal tunnel release using KnifeLight: evaluation of the safety and effectiveness of using a single wrist incision (cadaveric study). J Hand Surg Eur Vol. 2009;34:506- 10.

28. Croutzet P, Chassat R, Masmejean EH. Mini-invasive surgery for chronic exertional compartment syndrome of the forearm: a new technique. Tech Hand Up Extrem Surg. 2009; I 3: I 37-40.

29. Huisstede BM, Randsdorp MS, Coert JH, Glerum S, van Middelkoop M, Koes BW. Carpal tunnel syndrome. Part II: Effectiveness of surgical treatments. A systematic review. Arch Phys Med Rehabil. 2010;91:1005-24.

30. Varitimidis SE, Sotereanos DG. Vein Wrapping for the Treatment of Recurrent Entrapment Neuropathies En: Malizos K (Ed). Reconstructive Microsurgery. Georgetown: Landes Bioscience, 2003:25-3I.

31. Willbourn AJ. The electrodiagnostic examination with peripheral neve injuries. Clin Plast Surg. 2003;30: I 39-54. 